\title{
Hard diffraction at HERA in the dipole model of BFKL dynamics
}

\author{
S. Munier, R. Peschanski (SPhT) \\ Ch. Royon (DAPNIA/SPP) \\ Commissariat à l'Energie Atomique, Saclay, \\ F-91191 Gif-sur-Yvette Cedex \\ France
}

May 24, 2021

\begin{abstract}
Using the QCD dipole picture of the hard BFKL pomeron, we derive the general expressions of the elastic and inelastic components of the proton diffractive structure functions. We obtain a good 7 parameter fit (including a secondary reggeon contribution) to data taken at HERA by the $\mathrm{H} 1$ and ZEUS collaborations. The main characteristic features of the model in reproducing the data are discussed, namely the effective pomeron intercept, the scaling violations and the beta dependence. A difference obtained in the separate H1 and ZEUS fits leads us to analyse directly the differences between both measurements. Predictions on $\mathrm{R}$, the ratio of longitudinal to transverse photon cross sections are performed and lead to very large values at high beta and large virtuality $\mathrm{Q}$ which may lead to a discrimination between models.
\end{abstract}

\section{Introduction}

The HERA data for deep-inelastic scattering at high energy and high $Q^{2}$ contain a sizeable fraction of events in which one observes a large rapidity gap in the forward region [1], 2]. These events result from a colour-singlet exchange between the diffractively dissociated virtual photon and the proton. Since this phenomenon is present even for high virtuality of the photon, it is called hard diffraction, at variance with soft diffractive phenomena. Several theoretical formulations have been proposed [3]. Among the most popular models, the one based on a pointlike structure of the pomeron [1] has been studied quantitatively using a non-perturbative input supplemented by a DGLAP evolution [1]. In this formulation, it is assumed that the exchanged object, the pomeron, is a colour-singlet quasi-particle whose structure is probed in deep-inelastic scattering.

There exists a different approach [5, 6] in which the cross sections are determined by the interaction between colour dipole states [7] describing the photon and the proton. Indeed, it is well-known that the photon can be analyzed in terms of $q \bar{q}$ configurations [8] while it 
has been shown [9] that the small- $x$ structure function of the proton can be described by a collection of primordial dipoles with subsequent perturbative QCD evolution. More specifically [6], the combination of the dipole description of perturbative QCD at high energy and the Good-Walker mechanism [10] leads to a unified description of the proton total and diffractive structure functions [11]. However, a quantitative description of the data following [6] was still lacking. The aim of the present paper is to provide a simple and quantitative formulation fitting the experimental data.

In the dipole approach, two components are shown to contribute to the diffractive structure function (see fig.1). First, an elastic component (fig.1-a) corresponds to the elastic interaction of two dipole configurations. It is expected to be dominant in the finite $\beta$ region, i.e. for small relative masses of the diffractive system. Second, there is an inelastic component (fig.1-b) where the initial photon dipole configuration is diffractively dissociated in multi-dipole states by the target. This process is expected to be important at small $\beta$ (large masses). Note that both components are obtained in ref. [6] through perturbative QCD resummation corresponding to the BFKL approach [12]. In these respects, it is based on a "hard pomeron" approach at variance with e.g. a non perturbative pomeron input evolved using DGLAP evolution [1].

We present here fits of the published diffractive data [1], 2] based on a simple with 7 free parameters and analytic parametrization for the diffractive structure functions in the QCD dipole model. Both fits are successful showing that this approach is a good candidate for an understanding of hard diffraction. The main input of the calculation is a formulation of the elastic QCD amplitude $T\left(r, \rho, b ; x_{\mathbb{P}}\right)$ for the diffusion of a dipole of size $r$ on a dipole of size $\rho$ with rapidity gap $\log 1 / x_{\mathbb{P}}$ at fixed impact parameter $b$. This $b$-dependent input allows one to factorize the different integrals involved in the perturbative calculation of the diffractive components. The dipole model for the proton [9] is used to provide predictions for the HERA data. We also include a phenomenological secondary Regge trajectory which is known to play a rôle in the limited domain of large mass and small rapidity gap [1].

The outline of the paper is as follows: in section $\mathbf{2}$, we introduce the dipole formalism and in particular the input elastic dipole-dipole amplitude $T\left(r, \rho, b ; x_{\mathbb{P}}\right)$ following the requirements of the BFKL dynamics. Using this amplitude, we derive the general expressions for the elastic $(2.9,2.10)$, and inelastic (2.15) diffractive components for both transverse and longitudinal polarizations of the photon. In section $\mathbf{3}$ we obtain an analytical form of the proton diffractive amplitudes used to fit the HERA data. In section 4, the resulting fit is displayed. The simple form of the amplitudes makes transparent the discussion of the model properties e.g. the relative contribution of the two components, the effective exponent for the rapidity gap dependence, the origin of the scaling violations, and the $\beta$ dependence. Conclusions and outlook are given in the final section $\mathbf{5}$.

\section{The QCD dipole formalism}

As mentionned in the introduction, the main input of our approach to the structure functions is the interaction amplitude $T\left(r, \rho, b ; x_{\mathbb{P}}\right)$ for a dipole of size $\rho$ with a dipole of size $r$, at impact parameter $b$ where $\log 1 / x_{\mathbb{P}}$ is the rapidity gap. The knowledge of the amplitude as a function of $b$ is essential for the calculation of the two components of hard diffraction [6]. It is thus compulsory to start with a correct BFKL amplitude in the whole impact parameter space which goes beyond the commonly used expression for the forward elastic amplitude $T^{(\text {forward })}=\int d^{2} b T\left(r, \rho, b ; x_{\mathbb{P}}\right)$. For this sake, one uses the conformal invariance of the BFKL 
kernel in transverse coordinates [13]. We shall propose such a $b$-dependent amplitude by asking for the two following requirements:

(i) The integral over impact parameter space boils down to the known exact expression for the forward amplitude $\left.T^{\text {(forward) }} \llbracket 12,13\right]$.

(ii) It has the correct high impact parameter approximation [7] including the modification by a scale factor which has been deduced from conformal invariance [13.

Using the inverse-Mellin transform which appears in the solution of the BFKL equations [12], the amplitude reads:

$$
T\left(r, \rho, b ; x_{\mathbb{P}}\right)=\frac{1}{2} \frac{\rho^{2} r^{2}}{b^{4}} \int \frac{d \gamma}{2 i \pi}(1-2 \gamma) d(\gamma)\left(\frac{16 b^{2}}{r \rho}\right)^{2 \gamma} x_{\mathbb{P}}^{-\Delta_{\mathbb{P}}(\gamma)},
$$

where the factor 16 is the scale factor determined by conformal invariance.

$$
\Delta_{\mathbb{P}}(\gamma)=\frac{\alpha_{s} N_{c}}{\pi} \chi(\gamma) ; \chi(\gamma)=2 \psi(1)-\psi(\gamma)-\psi(1-\gamma)
$$

is the $\gamma$-dependent QCD pomeron intercept [12], and

$$
d(\gamma)=\frac{\alpha_{s}^{2}}{16 \gamma^{2}(1-\gamma)^{2}}
$$

is the two-gluon exchange elementary dipole-dipole cross section. As already mentioned, the amplitude meets the abovementionned requirements, since:

(i) the integration over $b$ leads to the known expression for the forward amplitude (for $r>\rho$ ):

$$
\int_{r / 4}^{+\infty} \int_{r / 4}^{+\infty} 2 \pi b d b T\left(r, \rho, b ; x_{\mathbb{P}}\right)=8 \pi \rho^{2} \int \frac{d \gamma}{2 i \pi} d(\gamma)\left(\frac{r}{\rho}\right)^{2 \gamma} x_{\mathbb{P}}^{-\Delta_{\mathbb{P}}(\gamma)} \equiv T^{(\text {forward })} .
$$

Note that the lower integration bound $r / 4$ on $b$ is required to recover the correct expression for the forward amplitude. In the absence of an exact conformal invariant solution for all impact parameter [14], this cutoff will play an important role in the determination of the hard diffraction components.

(ii) A saddle point approximation of the integral over $\gamma$ in formula (2.1) gives the approximate expressions in the large $b$ approximation used in ref. [6, 7], up to the scale factor 16. This factor is strictly speaking not present in the large $b$ approximation, but appears when one takes into account the global conformal invariance of the BFKL kernel [13. This scale factor leads to a much more central impact parameter distribution than the approximate expressions used in ref.[6, 7], and plays a major rôle in diffraction, as we shall see in the following.

Using formula (2.1) as an input leads to an analytic formulation of the diffractive amplitudes. In this section, we shall first consider hard diffraction on a dipole of given size $r$. It will be shown in the next section how, starting from this case, it is possible to derive phenomenological expressions for hard diffraction on a proton. One has the following identities for the diffractive structure functions [6]:

$$
F_{T, L}^{D}\left(Q^{2}, x_{\mathbb{P}}, M^{2}\right)=\frac{Q^{4}}{4 \pi^{2} \alpha_{e m}} \frac{1}{\beta x_{\mathbb{P}}} \frac{d \sigma_{T, L}}{d M^{2}}=\frac{Q^{2}}{4 \pi^{2} \alpha_{e m}} \frac{1}{x_{\mathbb{P}}} \beta \frac{d \sigma_{T, L}}{d \beta},
$$

where $d \sigma_{T}$ (resp. $d \sigma_{L}$ ) is the differential cross section for the scattering of a virtual photon with transverse (resp. longitudinal) polarization, on a dipole. 


\section{a) Elastic component}

Using the QCD dipole model for the elastic component (see fig. 1.a), one writes

$$
\frac{d \sigma_{T, L}^{(e l)}}{d M^{2}}=\frac{N_{c} \alpha_{e m} e^{2}}{2 \pi} Q^{2} \int_{r / 4}^{\infty} d^{2} b \int_{0}^{1} d z f_{T, L}(z)\left|\mathbb{T}_{T, L}\left(b ; x_{\mathbb{P}}\right)\right|^{2}
$$

where

$$
\mathbb{T}_{T, L}\left(b ; x_{\mathbb{P}}\right)=\int_{0}^{4 b} d \rho \rho T\left(r, \rho, b ; x_{\mathbb{P}}\right) K_{1,0}(\hat{Q} \rho) J_{1,0}(\hat{M} \rho)
$$

and

$$
\begin{aligned}
& f_{T}(z) \equiv z^{2}(1-z)^{2}\left(z^{2}+(1-z)^{2}\right) \\
& f_{L}(z) \equiv 4 z^{3}(1-z)^{3} .
\end{aligned}
$$

The notation " $\hat{X}$ " is defined by $\hat{X}=(z(1-z))^{1 / 2} X$.

Following ref. [6], the formulae (2.6-2.8) describe the interaction of a $q \bar{q}$ configuration of transverse size $\rho$ of the virtual photon with a dipole of size $r$. The input elastic amplitude is taken from (2.1). The photon wave function in terms of $q \bar{q}$ configurations is projected on its transverse $(T)$ and longitudinal $(L)$ polarization states which differ only by the factors $f_{T, L}(z)$ and by the order of the Bessel functions [15] involved: $K_{1}, J_{1}$ are associated to the transverse component, and $K_{0}, J_{0}$ to the longitudinal one. $z$ (resp. $\left.(1-z)\right)$ is the longitudinal momentum fraction carried by the antiquark (resp. quark). Note that interference effects are present [6] which explain the product of Bessel functions of different kinds $(K$ and $J)$. The cutoff in impact parameter space appears twice in formulae (2.6) and in (2.7), first by the upper bound $4 b$ on $\rho$

and second by a lower bound $r / 4$ on the impact parameter $b$. In practice, we will release the first bound, which will greatly simplify the calculations and allow to find an analytical form for the diffractive amplitude. This approximation is expected to change only the normalisations.

Inserting expression (2.1) for the dipole-dipole amplitude in eq. (2.6, 2.7), one obtains the results for $F_{T, L}^{D}$ (2.5) involving integrals over the impact parameter $b$, the momentum fraction $z$ and the $q \bar{q}$-pair size $\rho$. Interestingly enough, these integrations can be factorized and exactly computed using in particular the change of variable $\rho \rightarrow \hat{\rho}=(z(1-z))^{1 / 2} \rho$. After a tedious but straightforward calculation using well-known identities [15], the successive integrations give

$$
\begin{aligned}
F_{T}^{D(e l)}=\frac{16^{3}}{2 x_{\mathbb{P}}} \frac{N_{c} e^{2}}{\pi^{2}} \beta^{2}(1-\beta)\left(\frac{Q_{0}}{Q}\right)^{2} \int \prod_{j=1,2}\left[\frac{d \gamma_{j}}{2 i \pi}\left(\frac{Q}{2 Q_{0} \sqrt{\beta}}\right)^{2 \gamma_{j}} x_{\mathbb{P}}^{-\Delta_{\mathbb{P}}\left(\gamma_{j}\right)}\right] \\
\times \prod_{j=1,2}\left\{\left(1-2 \gamma_{j}\right) d\left(\gamma_{j}\right) \Gamma\left(2-\gamma_{j}\right) \Gamma\left(3-\gamma_{j}\right)_{2} F_{1}\left(2-\gamma_{j},-1+\gamma_{j} ; 2 ; 1-\beta\right)\right\} \\
\times \frac{\gamma_{1}+\gamma_{2}}{\left(\gamma_{1}+\gamma_{2}-1\right)\left(3-2\left(\gamma_{1}+\gamma_{2}\right)\right)} B\left(\gamma_{1}+\gamma_{2}, 1 / 2\right), \\
F_{L}^{D(e l)}=\frac{16^{3}}{x_{\mathbb{P}}} \frac{N_{c} e^{2}}{\pi^{2}} \beta^{3}\left(\frac{Q_{0}}{Q}\right)^{2} \int \prod_{j=1,2}\left[\frac{d \gamma_{j}}{2 i \pi}\left(\frac{Q}{2 Q_{0} \sqrt{\beta}}\right)^{2 \gamma_{j}} x_{\mathbb{P}}^{-\Delta_{\mathbb{P}}\left(\gamma_{j}\right)}\right] \\
\times \prod_{j=1,2}\left\{\left(1-2 \gamma_{j}\right) d\left(\gamma_{j}\right) \Gamma^{2}\left(2-\gamma_{j}\right){ }_{2} F_{1}\left(2-\gamma_{j},-1+\gamma_{j} ; 1 ; 1-\beta\right)\right\} \\
\times \frac{1}{3-2\left(\gamma_{1}+\gamma_{2}\right)} B\left(\gamma_{1}+\gamma_{2}, 1 / 2\right),
\end{aligned}
$$


where we have introduced the scale $Q_{0} \equiv 2 / r$. Note that these expressions involve two coupled inverse-Mellin transforms in $\gamma_{1}$ and $\gamma_{2}$, each corresponding to one elastic dipole interaction, (see fig.1-a).

\section{b) Inelastic component}

As shown in fig. 1.b, the inelastic component stems from the following process: the initial $q \bar{q}$ state of the virtual photon develops a set of colour dipoles through cascading [7] and the diffractive component is due to the interactions of two of the produced dipoles with a target dipole, each of these being described by the amplitude (2.1). The cross section can be expressed as [6]:

$$
\beta \frac{d \sigma_{T, L}^{(i n)}}{d \beta}=16^{3} \alpha_{e m} e^{2} \alpha_{s}^{5} N_{c}^{2} \frac{1}{Q^{2}} \int_{r / 4}^{\infty} \frac{d^{2} b}{b^{2}}\left\langle\varrho^{2}\right\rangle^{2} \int \frac{d \gamma}{2 i \pi}\left(\frac{b Q}{2}\right)^{2 \gamma}(1-\gamma)^{3} H_{T, L}(\gamma) \beta^{-\Delta_{\mathbb{P}}(\gamma)}
$$

where

$$
\left\langle\varrho^{2}\right\rangle=\int_{0}^{4 b} \frac{d \varrho}{\varrho} \varrho^{2} \frac{T\left(r, \varrho, b ; x_{\mathbb{P}}\right)}{4 \pi \alpha_{s}^{2} \varrho^{2}}
$$

and

$$
\begin{aligned}
H_{T}(\gamma) & =\frac{V(\gamma)}{16 \gamma^{2}(1-\gamma)^{4}} \frac{\Gamma(3-\gamma) \Gamma^{3}(2-\gamma) \Gamma(2+\gamma) \Gamma(1+\gamma)}{\Gamma(4-2 \gamma) \Gamma(2+2 \gamma)} \\
H_{L}(\gamma) & =\frac{V(\gamma)}{8 \gamma(1-\gamma)^{3}(1+\gamma)(2-\gamma)} \frac{\Gamma(3-\gamma) \Gamma^{3}(2-\gamma) \Gamma(2+\gamma) \Gamma(1+\gamma)}{\Gamma(4-2 \gamma) \Gamma(2+2 \gamma)}
\end{aligned}
$$

with

$$
V(\gamma)={ }_{3} F_{2}(1-\gamma, 1-\gamma ; 1,3 / 2 ; 1)
$$

The dipole-dipole interaction is represented by formula (2.12) which can be interpreted as the average squared transverse sizes of the dipoles seen at the impact parameter $b$. Indeed, the quantity $T\left(r, \varrho, b ; x_{\mathbb{P}}\right) / 4 \pi \alpha_{s}^{2} \varrho^{2}$ is nothing else than the density of dipoles of size $\varrho$ emitted from a dipole of size $r$ at impact parameter $b$ [7, 13]. The inverse Mellin-transform and its integrand factor $V(\gamma)$ (2.14) comes from the calculation of the $1 \rightarrow 2$ dipole branching at the virtual

photon vertex [6]. The different prefactors appearing in $H_{T}$ and $H_{L}$ in formula (2.13) come from the transverse and longitudinal wave-functions of the virtual photon, respectively.

Inserting the expression (2.12) into eq. (2.11) and integrating over the impact parameter $b$, one gets:

$$
\begin{aligned}
& F_{T, L}^{D(i n)}=\frac{1}{x_{\mathbb{P}}} \frac{16^{5}}{\pi^{3}} e^{2} \alpha_{s} N_{c}^{2} \int \prod_{j=1,2}\left\{\frac{d \gamma_{j}}{2 i \pi} \frac{1-2 \gamma_{j}}{2-2 \gamma_{j}} d\left(\gamma_{j}\right) x_{\mathbb{P}}^{-\Delta_{\mathbb{P}}\left(\gamma_{j}\right)}\right\} \\
& \times \int \frac{d \gamma}{2 i \pi}\left(\frac{Q}{4 Q_{0}}\right)^{2 \gamma} \beta^{-\Delta_{\mathbb{P}}(\gamma)}(1-\gamma)^{3} H_{T, L}(\gamma) \frac{1}{2-\gamma-\gamma_{1}-\gamma_{2}} .
\end{aligned}
$$

The formulae $(2.9,2.10,2.15)$ condense in an analytical form the perturbative QCD predictions for the diffractive structure functions of a dipole of size $2 / Q_{0}$. 


\section{Proton diffractive structure functions in the dipole model}

In the previous section, we have obtained the expressions for the diffractive structure functions, when the target is a single dipole of size $r=2 / Q_{0}$. Our aim in this section is to use these formulae to obtain the diffractive structure functions of the proton. We have thus to implement a model for the proton which could describe it as a collection of primordial dipoles.

\section{a) Elastic component}

Applying the steepest-descent method, the saddle-point is determined by the usual BFKL-type integrand (between the square brackets in $(2.9,2.10))$. It reads:

$$
\gamma_{s p}=\frac{1}{2}\left(1-a\left(x_{\mathbb{P}}\right) \log \frac{Q}{2 Q_{0} \sqrt{\beta}}\right) .
$$

where $a(x)=\pi /\left(7 \zeta(3) \alpha_{s} N_{c} \log 1 / x\right)$ is the $k_{\perp}$-diffusion coefficient coming from the BFKL evolution [12, 16]. We have to use a special treatment for the pole at $\gamma_{1}+\gamma_{2}=1$ in formula (2.9), since it contributes for the asymptotic saddle points at $\gamma_{1}=\gamma_{2}=1 / 2$ when $x_{\mathbb{P}} \rightarrow 0$. All the other singularities are not relevant, since they are far from the saddle points.

Noting that in the kinematical domain of interest, $1-2 \gamma_{s p}=a\left(x_{\mathbb{P}}\right) \log Q / 2 Q_{0} \sqrt{\beta} \ll 1$, and working at lowest order, the results are:

$$
\begin{aligned}
F_{T}^{D(e l)}=12 \frac{N_{c} e^{2} \alpha_{s}^{4}}{\pi} x_{\mathbb{P}}^{-2 \alpha_{\mathbb{P}}+1} a^{3}\left(x_{\mathbb{P}}\right) \log ^{3} \frac{Q}{2 Q_{0} \sqrt{\beta}} e^{-a\left(x_{\mathbb{P}}\right) \log ^{2} \frac{Q}{2 Q_{0} \sqrt{\beta}}} & \\
& \times \beta(1-\beta)\left[{ }_{2} F_{1}\left(-\frac{1}{2}, \frac{3}{2} ; 2 ; 1-\beta\right)\right]^{2}, \\
F_{L}^{D(e l)}=16 \frac{N_{c} e^{2} \alpha_{s}^{4}}{\pi} x_{\mathbb{P}}^{-2 \alpha_{\mathbb{P}}+1} a^{3}\left(x_{\mathbb{P}}\right) \log ^{2} \frac{Q}{2 Q_{0} \sqrt{\beta}} e^{-a\left(x_{\mathbb{P}}\right) \log ^{2} \frac{Q}{2 Q_{0} \sqrt{\beta}}} & \times \beta^{2}\left[{ }_{2} F_{1}\left(-\frac{1}{2}, \frac{3}{2} ; 1 ; 1-\beta\right)\right]^{2},
\end{aligned}
$$

with

$$
\alpha_{\mathbb{P}} \equiv 1+\Delta_{\mathbb{P}}\left(\frac{1}{2}\right)=1+\frac{\alpha_{s} N_{c}}{\pi} 4 \log 2 .
$$

The pole at $\gamma_{1}+\gamma_{2}=1$ in $F_{T}^{D(e l)}$ results in an extra $\log Q / 2 Q_{0} \sqrt{\beta}$ which may be large.

The formulae $(3.17,3.18)$ give an explicit expression for the elastic components of diffraction in the QCD dipole model. The $x_{\mathbb{P}}$-dependent prefactor $x_{\mathbb{P}}^{-2 \alpha \mathbb{P}+1} a^{3}\left(x_{\mathbb{P}}\right)$ plays the role of the (hard) pomeron flux factor already discussed in references [6, 11]. Revealing new features of the model, the present explicit calculation shows that the relevant scale factor responsible for the scaling violations is $2 Q_{0} \sqrt{\beta}$ where $Q_{0}$ is the scale of the proton-dipole amplitude. The $\beta$-dependence is found to be non polynomial.

\section{b) Inelastic component}

Using again the steepest-descent method for evaluating the three integrals in $\gamma, \gamma_{1}$ and $\gamma_{2}$ of formula (2.15), we find saddle-points at

$$
\gamma_{s p}=\frac{1}{2}\left(1-a(\beta) \log \frac{Q}{4 Q_{0}}\right), \gamma_{j, s p}=\frac{1}{2}\left(1-a\left(x_{\mathbb{P}}\right)\right) .
$$


Note that there is no coupling of the integrals at the saddle points; however, one should take into account the prefactor zeros at $\gamma_{j}=1 / 2$, see eq. (2.15). All in all, the result reads:

$$
F_{T, L}^{D(i n)}=2^{9} \sqrt{\frac{2}{\pi}} H_{T, L}\left(\frac{1}{2}\right) \frac{N_{c} e^{2} \alpha_{s}^{5}}{\pi^{4}} x_{\mathbb{P}}^{-2 \alpha_{\mathbb{P}}+1} a^{3}\left(x_{\mathbb{P}}\right) \frac{Q}{Q_{0}} e^{-\frac{a(\beta)}{2} \log ^{2} \frac{Q}{4 Q_{0}}} a^{\frac{1}{2}}(\beta) \beta^{-\Delta_{\mathbb{P}}} .
$$

Comparing our expression (3.21) with the original result (formula (11) in the second paper of ref.[6]), we find identical results but for the scale of the transverse momentum in the BFKL evolution: $4 Q_{0}$ instead of $Q_{0}$. This change comes from the scale factor determined by conformal invariance (see 2.1) and was not considered in the previous approximation [6]. This modified scale factor has an important phenomenological effect.

In the QCD dipole model, there is a tight connection between the total and diffractive structure functions. In refs. [9, 11], the QCD dipole model has been successfully applied to the proton total structure function in the small- $x$ kinematical domain. A model for the primordial dipole configurations in the proton has been introduced through a distribution with average size and the non perturbative features of the proton target manifest themselves only through this scale and the global normalization constant related to the density of primordial dipoles (for a more complete discussion, see ref.[9]). We thus use this representation of the nucleon target in formulae $(3.17,3.18,3.21)$ to derive the model for the proton diffractive structure functions. In this context, the scale parameter is reinterpreted as $Q_{0}=2 /\langle r\rangle$ and we introduce the arbitrary normalizations $N_{T}^{(e l)}, N_{L}^{(e l)}, N^{(i n)}$ for $F_{T, L}^{D(e l)}$ and $F_{T, L}^{D(i n)}$ multiplying formulae (3.17, 3.18 , 3.21) respectively. These unknown normalizations reflect the non-perturbative primordial dipole configurations contributing to the various proton structure functions.

With this model, we are ready to write a full parametrization adequate for the description of the data. The free parameters of the dipole model are $\alpha_{\mathbb{P}}$, which is related to the fixed coupling constant $\alpha_{s}$ in the BFKL scheme at leading order (see eq. (3.19)), $Q_{0}$, corresponding to a non-perturbative scale for the proton, and the three normalizations $N_{T}^{(e l)}, N_{L}^{(e l)}, N^{(i n)}$. As is now well-known, a secondary trajectory based on reggeon exchange is added in order to take into account the large-mass and small rapidity gap domain. Reggeon exchange can here be simply parametrized in the following way:

$$
F_{2}^{D(R)}\left(x_{P}, \beta, Q^{2}\right)=f^{\mathbb{R}}\left(x_{\mathbb{P}}\right) F_{2}^{\mathbb{R}}\left(\beta, Q^{2}\right),
$$

where the reggeon flux $f^{\mathbb{R}}\left(x_{\mathbb{P}}\right)$ is assumed to follow a Regge behaviour with a linear trajectory $\alpha_{\mathbb{R}}(t)=\alpha_{\mathbb{R}}(0)+\alpha_{\mathbb{R}}^{\prime} t:$

$$
f^{\mathbb{R}}\left(x_{\mathbb{P}}\right)=\int_{t_{c u t}}^{t_{\text {min }}} d t \frac{e^{B^{\mathbb{R}} t}}{x_{\mathbb{P}}^{2 \alpha_{\mathbb{R}}(t)-1}}
$$

where $\left|t_{\text {min }}\right|$ is the minimum kinematically allowed value of $|t|$ and $t_{c u t}=-1 \mathrm{GeV}^{2}$ is the limit of the measurement. The values of $B^{\mathbb{R}}$ and $\alpha_{\mathbb{R}}^{\prime}$ are fixed with data from hadron-hadron collisions [1]. The reggeon structure function is assumed to be the pion structure function [17]. The free parameters for this component are the reggeon normalisation $N^{\mathbb{R}}$ and exponent $\alpha_{\mathbb{R}}$. The final parametrization used for the fit is the sum of the three contributions detailed above $(3.17,3.18$, 3.21, 3.22, namely:

$$
F_{2}^{D(3)}=\frac{1}{N_{C} e^{2}}\left(N^{i n}\left(F_{T}^{D(i n)}+F_{L}^{D(i n)}\right)+N_{T}^{e l} F_{T}^{D(e l)}+N_{L}^{e l} F_{L}^{D(e l)}+N_{R} F_{2}^{\mathbb{R}}\right) .
$$

Only one parameter $\left(N^{i n}\right)$ is used for the normalisation of the inelastic component since $F_{L}^{D(i n)} / F_{T}^{D(i n)}=2 / 9$. 


\section{Phenomenology}

\subsection{Fits to the $\mathrm{H} 1$ and ZEUS data}

A fit to the recently published H1 [1] and ZEUS [2] diffractive structure function data is performed separately. The result of both fits is shown in Figure 2 for $\mathrm{H} 1$ and Figure 3 for ZEUS, and their parameters are given in Table 1. Let us comment the interesting features of the fits. The fit to the $\mathrm{H} 1$ data leads to a very good $\chi^{2}(1.17$ per degree of freedom with statistical errors only). The value of $\alpha_{\mathbb{R}}$ is consistent with the usual values found for secondary reggeon contributions if interference effects are taken into account $\mathbb{1}$. The value of $\alpha_{\mathbb{P}}$ is found to be consistent with the expected intercept for a hard BFKL pomeron [12]. This intercept is higher than the value obtained from the fit to the structure function $F_{2}$ [9]. $Q_{0}$ is a typical non perturbative scale for the proton and very close to the value obtained in the proton structure function fit. It should be noted that the scale $Q_{0}$ appears in a quite non trivial way as the virtuality in the inelastic component $\left(Q / 4 Q_{0}\right)$, and in the elastic one $\left(Q / 2 \sqrt{\beta} Q_{0}\right)$. The validity of these results can be checked by starting with two different values of $Q_{0}$ for each component and the result of the fit leads to same values within errors, with the same $\chi^{2}$. Furthermore, imposing a constant scale for the elastic component, i.e. $Q / 2 \sqrt{\beta} Q_{0} \rightarrow Q / Q_{0}$, leads to a very bad quality fit. The other features of this description will be discussed further on.

The fit to the ZEUS data leads to a worse $\chi^{2}\left(\chi^{2} /\right.$ dof $=1.95$ with statistical errors only). The result is shown in Figure 3 in continuous line, and by comparison, the dashed curve corresponds to the parameters obtained in the $\mathrm{H} 1$ fit. It can be noticed that a difference is clearly seen both in the low $Q^{2}$, low $\beta$ and the high $Q^{2}$, high $\beta$ bins, in opposite way, the H1 fit going from below to above the Zeus fit.

In order to investigate the origin of these differences, a direct comparison between ZEUS and $\mathrm{H} 1$ data has been performed [18]. The H1 data have been interpolated to the ZEUS closest bins in $\beta$ and $Q^{2}$ using the dipole model fit. This interpolation is weakly sensitive to the model used as the interpolation in the kinematical variables is very small. It was checked that the use of the model by Bartels et al. [19] gives a similar result. The result of this comparison is displayed in Figure 4. The striking feature is that the main difference between both fits noticed in Figure 3 comes from the region where the data are most different. The differences occur both at small and high $\beta$ (i.e. small and high masses) and the solution of this puzzle cannot be due only to the difference in selecting diffractive events - either the rapidity gap selection [1] or the $M_{X}$ substraction method [2] - in both experiments. In fact, only high-mass events could be affected by these different selections. A global shift of ZEUS data of about $15 \%$ would be possible as they do not correct for proton dissociation but the difference between H1 and ZEUS data is not only due to normalisation effects. Indeed, it is clear in Figure 4 that, depending on the values of $Q^{2}$ and $\beta, H 1$ points lie either higher or lower than ZEUS data. The analysis of new accumulated data in this kinematical region would help solving the experimental puzzle and would be of great interest. 


\subsection{Effective pomeron intercept}

Let us now study the dependence on $x_{\mathbb{P}}$ which is directly related to the rapidity gap dependence. One can define an effective pomeron intercept in the following way:

$$
\alpha_{\mathbb{P}}^{e f f}=\frac{1}{2}\left(\frac{d \ln F_{2}^{D}}{d \ln 1 / x_{\mathbb{P}}}+1\right)
$$

where the $t$ dependence is integrated out (the data are mainly at $t \sim 0$ ). This effective exponent can also be determinated for the inelastic and elastic components separately as a function of $x_{\mathbb{P}}$ (Figure 5 ) or $Q^{2}$ (Figure 6).

In Figure 5, The effective intercepts of both components and their sums have been compared to the soft pomeron intercept [20] (dashed line) and to the bare hard BFKL pomeron obtained in the fit $\left(\alpha_{\mathbb{P}}=1.395\right.$, cf. Table 1$)$. The range of obtained values sits essentially between these two limits except in the large $x_{\mathbb{P}}$ region $\left(x_{\mathbb{P}} \geq 10^{-2}\right)$. It is clearly not consistent with the soft pomeron value (1.08). It is as well much lower than the bare pomeron intercept [6, 11]. This can be explained by the large logarithmic corrections induced by the $a^{3}\left(x_{\mathbb{P}}\right)$ term, proportional to $\log ^{3}\left(1 / x_{\mathbb{P}}\right)$, present in both diffractive components (see formulae $3.17,3.18,3.21$ ). The effect of this logarithmic term induces also an $x_{\mathbb{P}}$ dependence of the intercept. Moreover, in Figure 5 , it can be seen that the $x_{\mathbb{P}}$ dependence of the intercept is different between the elastic and the inelastic components. This induces a breaking of factorisation directly for the diffractive components of this model, which comes in addition to the known factorisation breaking due to secondary trajectories. In addition, the elastic component itself does not factorise, as it can be seen in Figure 5 for different $Q^{2}$ values. This comes from the mixing of the $x_{\mathbb{P}}$ and $Q^{2}$ dependence in formula 3.17, 3.18.

As seen in Figure 6, the $Q^{2}$ dependence of the pomeron intercept (taken for instance at $x_{\mathbb{P}}=0.001$ where the reggeon contribution is negligible) is weak. It is in good agreement with the H1 determination $\left(\alpha_{\mathbb{P}}=1.204 \pm .02\right)$ indicated in Figure 6. It is thus intermediate between the soft and hard pomeron lines but inconsistent with both of them. In our model, this softening of the bare pomeron comes from the large logarithmic corrections which cannot be neglected in perturbative calculations as was mentioned in the last paragraph.

\subsection{Scaling violations}

One striking feature of the diffractive proton structure functions was the $Q^{2}$ dependance at fixed $x_{\mathbb{P}}$ as a function of $\beta$ as was pointed out experimentally by the $\mathrm{H} 1$ collaboration [1] (see Figure 7) and confirmed at lower $Q^{2}$ [21]. The structure functions are increasing with $Q^{2}$ even at very high $\beta$ (see Figure 7) at variance with the behaviour of the total proton structure function as a function of $x$. In the QCD dipole model, this experimental feature is described by a non trivial interplay between the two diffractive components. In Figure 7 , the dipole fit is compared with the $\mathrm{H} 1$ result showing the contribution of each component: at small $\beta$, the inelastic component dominated and vary quasi linearly in $\log Q^{2}$, and at high $\beta$, this component is depressed similarly to the total structure function, but is progressively substituted by the elastic component. Note that the enhancement present in the data at high $\beta$ and low $Q^{2}$ which is probably due to vector meson production is partially reproduced by the model. It is expected that including the specific vector meson contributions in addition to the elastic $q \bar{q}$ component will even improve the result of the model in this kinematical range. It is striking that it is 
possible to describe the observed scaling violations in a very different framework as the one given by the DGLAP evolution as was performed by the H1 collaboration [1].

\section{$4.4 \beta$-dependence}

In Figure 8 is displayed the $\beta$ dependence of the proton diffractive structure function for a fixed value of $x_{\mathbb{P}}=0.003$ for different values of $Q^{2}$. The $\beta$ dependence is quite weak in all $Q^{2}$ bins and correctly reproduced by the interplay of the two components of the QCD dipole model. While at low $Q^{2}$, this effect is essentially due to the inelastic component, at high $Q^{2}$ the interplay between both components is required to describe the observed $\beta$-dependence.

The interplay of the different components can be analysed in more details in Figure 9 where the elastic, inelastic, reggeon components and their sum are displayed for three different values of $x_{\mathbb{P}}$ and four different values of $Q^{2}$ as a function of $\beta$. The reggeon component is only important at high value of $x_{\mathbb{P}}$ as expected and dominates at low $\beta$. It disappears at smaller $x_{\mathbb{P}}$. At low $Q^{2}$, the inelastic component dominates in almost the full $\beta$ range, while at higher $Q^{2}$, it is only important at low $\beta$ and replaced at low masses by the inelastic component.

\subsection{Longitudinal contribution to the diffractive structure function}

In Figure 10 are displayed as a function of $\beta$ in different $Q^{2}, x_{\mathbb{P}}$ bins the longitudinal and transverse components of the proton diffractive structure functions. Note that the $x_{\mathbb{P}}$ values are chosen a bit smaller than in the previous figure to depress the reggeon contribution. Note also that we did not separate the longitudinal and the transverse contributions for the inelastic component as they are directly proportional by a factor $H_{L}(1 / 2) / H_{T}(1 / 2)=2 / 9$ (see formula 3.21). In addition to the already mentioned dominance of the inelastic component at small $Q^{2}$, the longitudinal elastic component is found to be high at high $\beta$ and crosses over the transverse component near $\beta \sim 0$.8. It is thus expected to obtain high values of the ratio $R$ of the longitudinal to the transverse components at high values of $\beta$. The result of our fit prediction is displayed in Figure 11. We note that the $R$ ratio remains small $(\sim 0.2)$ in almost the full kinematical plane except notably at high $\beta$ where it may reach high values such as 2 . Note that this value is in the range of the measured $R$ ratio with vector meson production [3]. A measurement of $R$ in diffraction would thus be of great interest and would be a good test of the model. The question arises whether such high values of $R$ would not modify the $F_{2}^{D}$ measurement itself as it was assumed to be 0 [1]. We have checked that the parameters and the quality of the fit are not much influenced if only data points with $y<0.3$, where the influence of $R$ is small, are taken.

It is instructive to notice that another model of diffraction based on selecting $q \bar{q}$ and $q \bar{q} g$ components of the photon 19 also leads to a large contribution of the longitudinal $q \bar{q}$ contribution at high $\beta$. However, a distinct feature of the dipole model is the above mentioned difference between the high and low $Q^{2}$ behaviour where at low $Q^{2}$, the inelastic component dominates and induces a small value of $R$. We thus expect thet a measurement of $R$ in diffraction at low $Q^{2}$ would be a way to distinguish both models. 


\section{Conclusion and outlook}

Let us summarize the results we have obtained. First of all, the colour dipole model has provided us with a interesting theoretical and phenomenological framework to study hard diffraction. On thetheoretical ground, it enables to interpret hard diffraction in terms of BFKL dynamics of (resummed) perturbative QCD, which allows one to make theoretical estimates. On the other hand, the success of the phenomenological fit using analytical amplitudes allow a discussion of the various interesting and intringuing aspects of the data.

The model predicts two contributions: an elastic one for which the final state consists in the hadronization of a quark-antiquark pair, and an inelastic one, including soft gluon radiation reproduced in terms of a cascading process involving colour dipoles. Both contribute in quite different kinematical domains since they are characterized by different mass distributions (different $\beta$ ) and for different photon polarization. In this paper, we have obtained a suitable parametrization and a satisfactory fit to the H1 data along these lines. The fit to Zeus data is less satisfactory and with different parameter values. By a model-independent analysis of both data, we could identify the reason of the discrepancy in a genuine difference between data sets when interpolating in the same bins. This point deserves a detailed experimental study in the near future, since it may be of importance to doiscriminate between models. in particular the confirmation of the trend of present $\mathrm{H} 1$ data would be in favor of the dipole model.

On the theoretical ground, we have computed amplitudes obeying some of the requirements of BFKL dynamics for diffractive amplitudes (on a dipole). However, exact calculations of these rather complicated amplitudes are still under way [22] and we hope in the near future obtain definite predictions with all requirements of BFKL dynamics. the evaluation of next-leading BFKL effects would also be welcome since they already play a rôle for the total structure functions.

Finally, as emphasized in ref. 11] the colour dipole model formalism calls for a unified description of the diffractive and total deep-inelastic scattering events, e.g. including events with no rapidity gap. We showed that within the precision of the current data, there are quite a few indications (similar scale $Q_{0}$, softening of the gard Pomeron by logarithmic facors in diffraction,etc...) of such a common theoretical ground. However, further tests of the model are deserving. The first one would be a confrontation of the predicted $R$ ratio with the data if available: indeed, the various models should predict quite different contributions from the two polarization states of the photon. Other useful tests concern the final states. For instance one can compute the predictions for diffractive vector meson production and confront them to the recent data. Such tests might help distinguish between the few different models for hard diffraction which are able to describe the data. definitely, the QCD dipole model of BFKL dynamics is one of them.

\section{Acknowledgements}

A fruitful collaboration with Andrzej Bialas and Henri Navelet which has much inspired the present paper is acknowledged. We also thank them for very useful physical and technical remarks and suggestions during the elaboration of the paper. 


\section{References}

[1] C. Adloff et al., H1 coll., Z.Phys. C76 (1997) 613.

[2] J. Breitweg, ZEUS coll., Eur. Phys. J. C1 (1998) 81.

[3] For a recent review, see: Proceedings of the DIS98 workshop, 4-8 April 1998, Brussels

[4] G. Ingelman, P. Schlein, Phys. Lett. B152 (1985) 256.

[5] For a related approach, see N. N. Nikolaev, B. G. Zakharov, Z. Phys. C49 (1991) 607; Z. Phys. C53 (1992) 331.

[6] Elastic component: A. Bialas, R. Peschanski, Phys. Lett. B387 (1996) 405. Inelastic component: A. Bialas, R. Peschanski, Phys. Lett. B378 (1996) 302.

[7] A. H. Mueller, Nucl. Phys. B415 (1994) 373;

A. H. Mueller, B. Patel, Nucl. Phys. B425 (1994) 471;

A. H. Mueller, Nucl. Phys. B437 (1995) 107.

[8] J. D. Bjorken, J. Kogut, D. Soper, Phys. Rev. D3 (1971) 1382.

[9] H. Navelet, R. Peschanski, Ch. Royon, S. Wallon, Phys. Lett. B385 (1996) 357.

S. Munier, R. Peschanski, Nucl. Phys. B524 (1998) 377.

[10] M. L. Good, W. D. Walker, Phys. Rev. 120 (1960) 1857.

[11] A. Bialas, R. Peschanski, Ch. Royon, Phys. Rev. D57 (1998) 6899.

[12] V. S. Fadin, E.A. Kuraev and L.N. Lipatov, Phys. Lett. B60 (1975) 50;

I. I. Balitsky and L.N. Lipatov, Sov. J. Nucl. Phys. 28 (1978) 822.

[13] G. Salam, Nucl. Phys. B461 (1995) 512.

H. Navelet, R. Peschanski, Nucl. Phys. B507 (1997) 353.

H. Navelet, S. Wallon, Nucl. Phys. B522 (1998) 237.

[14] S. Munier, H. Navelet, R. Peschanski, Ch. Royon, L. Schoeffel, A. Van Haecke, in preparation.

[15] P.S. Gradshteyn, I.M. Ryzhik, Table of integrals series and products, (Academic Press, New York and London, 1965).

A. P. Prudnikov, Yu. A. Brychkov, O. I. Marichev, Integrals and Series (vol. 3), Gordon and Breach science publishers, 1986.

[16] J. Bartels, H. Lötter, Phys. Lett. B309 (1993) 400.

[17] M.Glück, E.Reya, A.Vogt, Z.Phys. C53 (1992) 651.

[18] C.Royon, Contribution to the Zeuthen workshop on low-x physics, 3-6 June 1998, Berlin.

[19] J. Bartels, J. Ellis, H. Kowalski, M. Wüsthoff, hep-ph/9803497.// J.Bartels, C.Royon, in preparation. 
[20] A.Donnachie, P.V.Landshoff, Phys. Lett. B296 (1992) 227.

[21] C. Adloff et al., H1 coll., Contributed paper to the ICHEP 98 conference, 23-30 July 1998, Vancouver.

[22] A. Bialas, H. Navelet, R. Peschanski, Phys. Lett. B427 (1998) 147, and in progress. 


\section{TABLE CAPTION}

\section{Table I}

Parameters obtained for the $F_{2}^{D}$ fit.

The fit has been performed with statistical errors only. The first error quoted is the statistical one and the second the systematic one.

\begin{tabular}{|c|c|c|}
\hline & H1 & ZEUS \\
\hline \hline$\alpha_{P}$ & $1.395 \pm 0.005 \pm 0.003$ & $1.327 \pm 0.001 \pm 0.016$ \\
$\alpha_{R}$ & $0.682 \pm 0.046 \pm 0.049$ & - \\
$Q_{0}$ & $0.428 \pm 0.011 \pm 0.001$ & $0.241 \pm 0.002 \pm 0.014$ \\
\hline$N^{\text {in }}$ & $0.00244 \pm 0.00028 \pm 0.00033$ & $0.00374 \pm 0.00015 \pm 0.00076$ \\
$N_{T}^{e l}$ & $40.0 \pm 1.6 \pm 3.6$ & $126.9 \pm 0.5 \pm 37.6$ \\
$N_{L}^{e l}$ & $12.8 \pm 1.2 \pm 1.1$ & $24.43 \pm 0.51 \pm 8.12$ \\
$N_{R}$ & $7.44 \pm 1.84 \pm 3.50$ & $0 \pm 58 \pm 0$ \\
\hline$\chi^{2}$ & 255.4 for 226 pts & 89.6 for 53 pts \\
& $1.17 /$ d.o.f. & $1.95 /$ d.o.f. \\
\hline
\end{tabular}

Table I 


\section{FIGURE CAPTIONS}

Figure 1 Schematic description of the QCD dipole model of diffraction. 1-a: elastic component, 1-b: inelastic component

Figure 2 Result of the $F_{2}^{D}$ fit to the H1 data [ []]. The data are displayed by triangles (with statistical and systematic errors added in quadrature) as a function of $x_{\mathbb{P}}$ in $\beta$ and $Q^{2}$ bins. The fit has been performed with statistical errors only and is displayed in full line (see text).

Figure 3 Result of the $F_{2}^{D}$ fit to the ZEUS data [2]. The data are displayed by triangles with statistical and systematic errors added in quadrature as a function of $x_{\mathbb{P}}$ in $\beta$ and $Q^{2}$ bins. The fit has been performed with statistical errors only and is displayed in full line (see text). For comparison, he result of the fit of the $\mathrm{H} 1 F_{2}^{D}$ data is displayed in dashed line.

Figure 4 Direct comparison between $H 1$ (squares) and ZEUS (triangles) data. The H1 data have been interpolated to the ZEUS bins by using the dipole parametrisation. Both data are found compatible within error bars. Note however that sensitive differences are seen in particular in three different $\beta$ and $Q^{2}$ bins $\left(Q^{2}=8 \mathrm{GeV}^{2}\right.$ for $\beta=0.2, Q^{2}=60 \mathrm{GeV}^{2}$, for $\beta=0.7$ and 0.9) (see text).

Figure 5 Effective pomeron intercept as a function of $x_{\mathbb{P}}$. The dependence of the effective pomeron intercept $\alpha_{\mathbb{P}}$ on $x_{\mathbb{P}}$ is shown for the inelastic component (independent of $Q^{2}$; continuous line) and for the inelastic one for 3 different values of $Q^{2}$. The value of $\beta$ is fixed at 0.3. The straight lines at $\alpha_{\mathbb{P}}=1.395$ and $\alpha_{\mathbb{P}}=1.08$ correspond respectively to the bare BFKL intercept as found by the fit (see table 1), and the soft Donnachie Landshoff prediction [20].

Figure 6 Effective pomeron intercept as a function of $Q^{2}$. The effective pomeron intercept of the model is displayed: elastic component (dashed curve), inelastic component (dotted curve), total (full curve). The value of $\beta$ and $x_{\mathbb{P}}$ are fixed for reference at $\left(\beta=0.3\right.$ and $\left.x_{\mathbb{P}}=10^{-3}\right)$. The bare and soft pomeron intercepts are given by dotted-dashed straight lines. The H1 determination [1] lies in the region between the two dashed straight lines.

Figure 7 Scaling violations. The dependence of $x_{\mathbb{P}} F_{2}^{D}$ on $Q^{2}$ for different values of $\beta$ at fixed $x_{\mathbb{P}}\left(3.10^{-3}\right)$ is shown together with the dipole model fit. Dotted line: elastic component, dashed line: inelastic component, full line: total. 
Figure 8 Beta dependence. The dependence of $x_{\mathbb{P}} F_{2}^{D}$ on $\beta$ for different values of $Q^{2}$ at fixed $x_{\mathbb{P}}$ $\left(3.10^{-3}\right)$ is shown together with the dipole model fit. Dotted line: elastic component, dashed line: inelastic component, full line: total.

Figure 9 Different components of the $\mathrm{H}_{1} \mathrm{~F}_{2}^{D}$ fit. Dotted line: elastic component, dashed line: inelastic component, dashed-dotted line: secondary reggeon component, full line: sum of all components.

Figure 10 Different components of the $H 1 F_{2}^{D}$ fit. Longitudinal versus transverse. Dotted line: tranverse elastic component, dotted-dashed line: longitudinal elastic component, dashed line: total $\left(F_{T}+F_{L}\right.$ with $F_{L} / F_{T}=2 / 9$, see text) inelastic component, full line: result of the fit.

Figure 11 Prediction for $R$, the ratio of the longitudinal to the transverse cross section. Note the different scale for the high $\beta$ bins. 


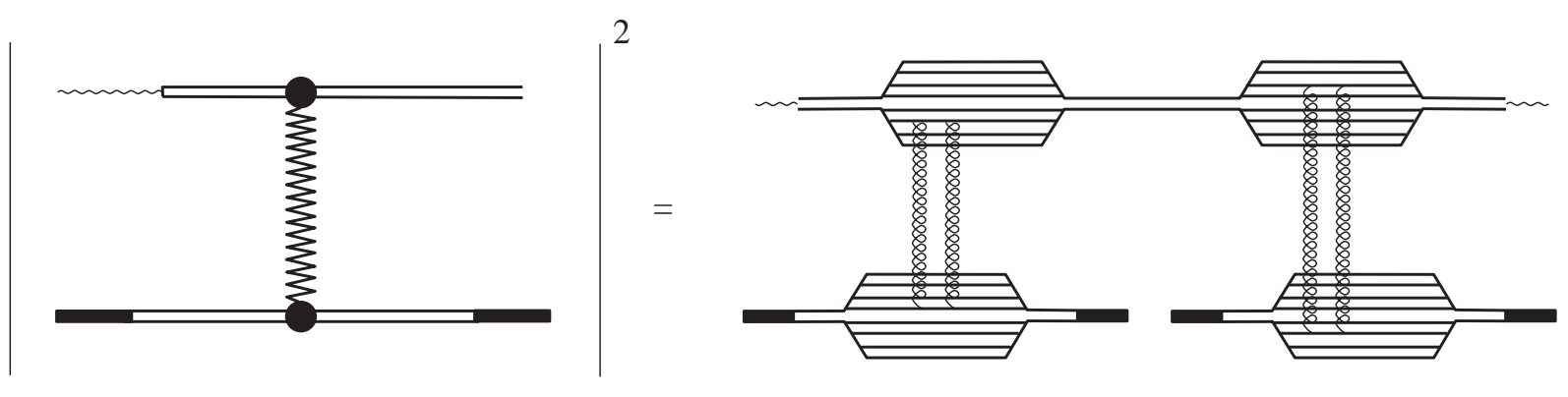

a.

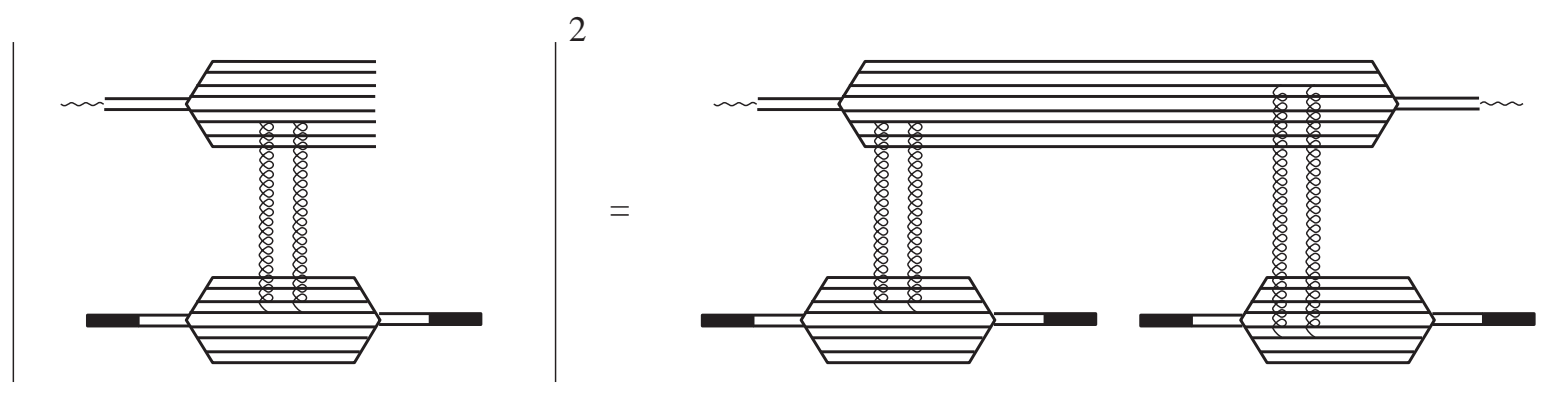

b.

Figure 1 


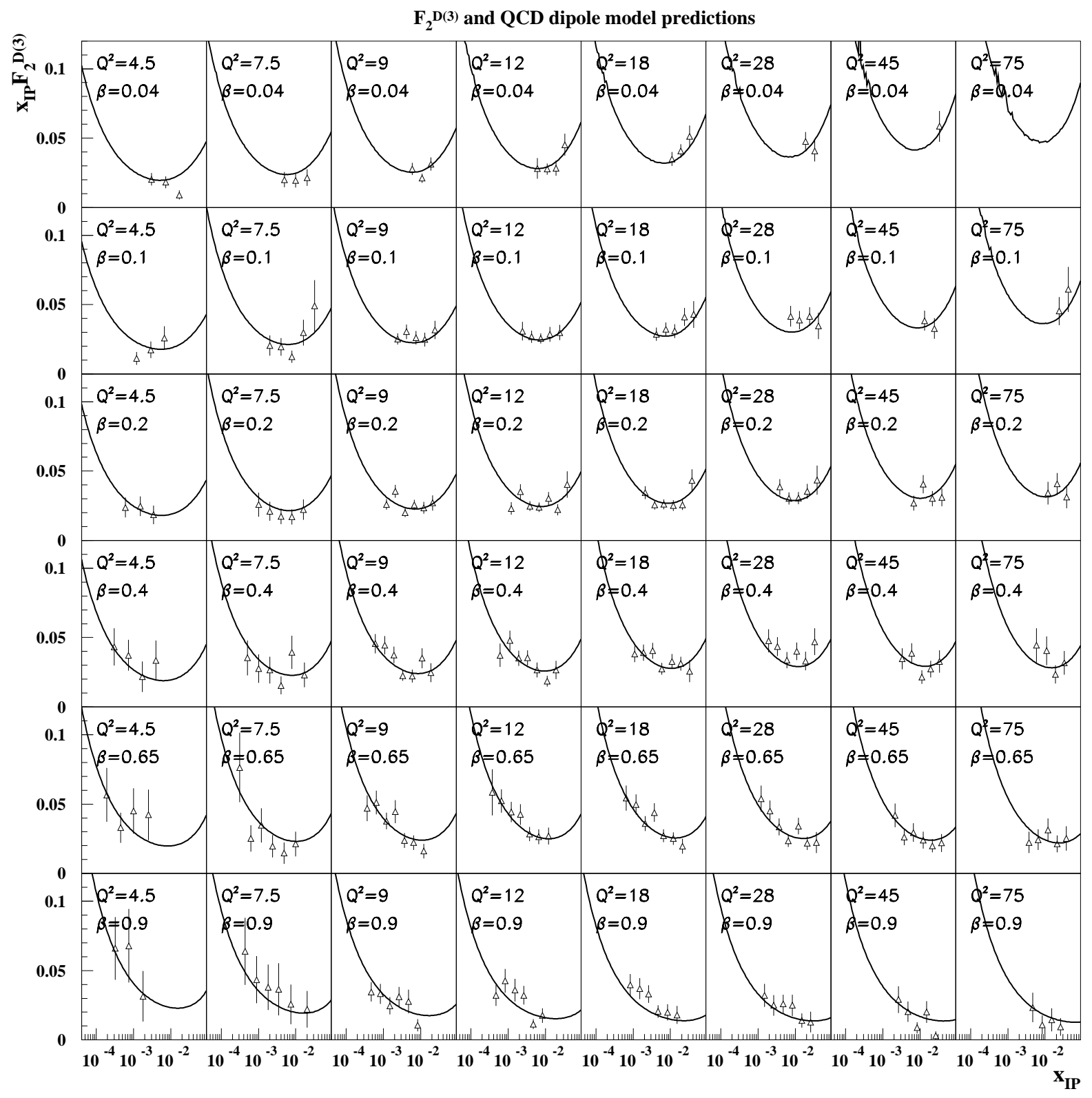

Figure 2 


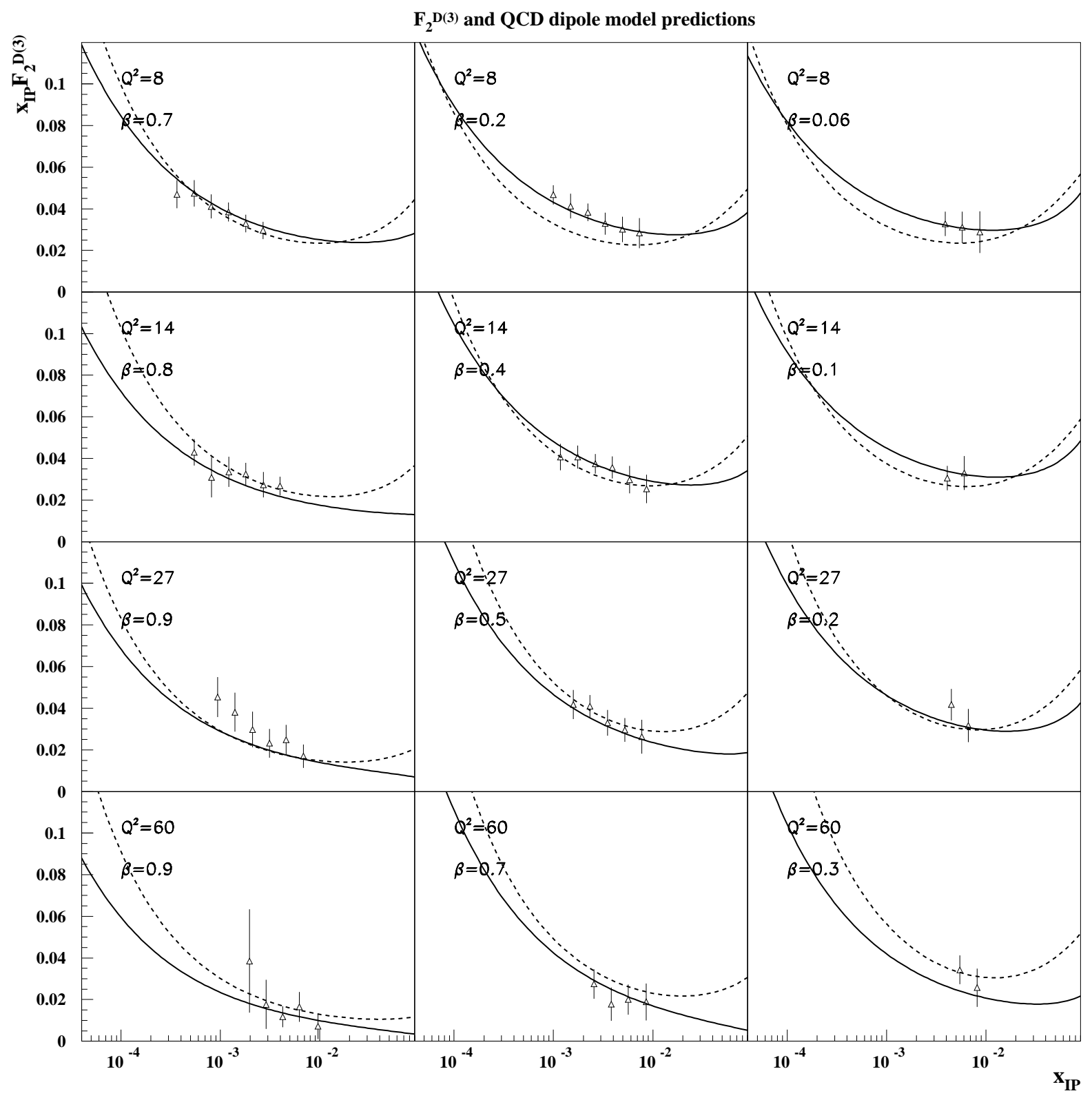

Figure 3 


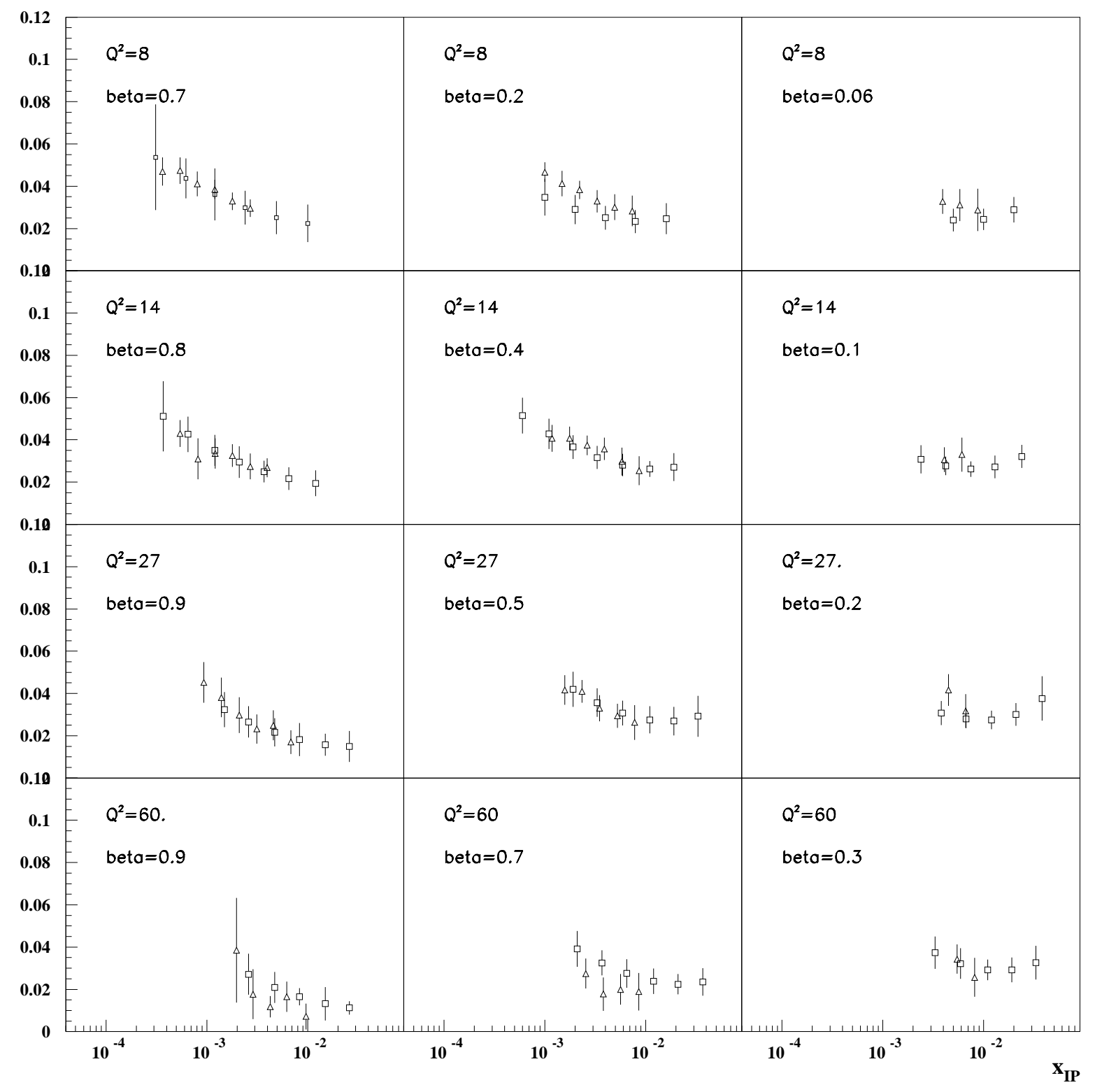

Figure 4 


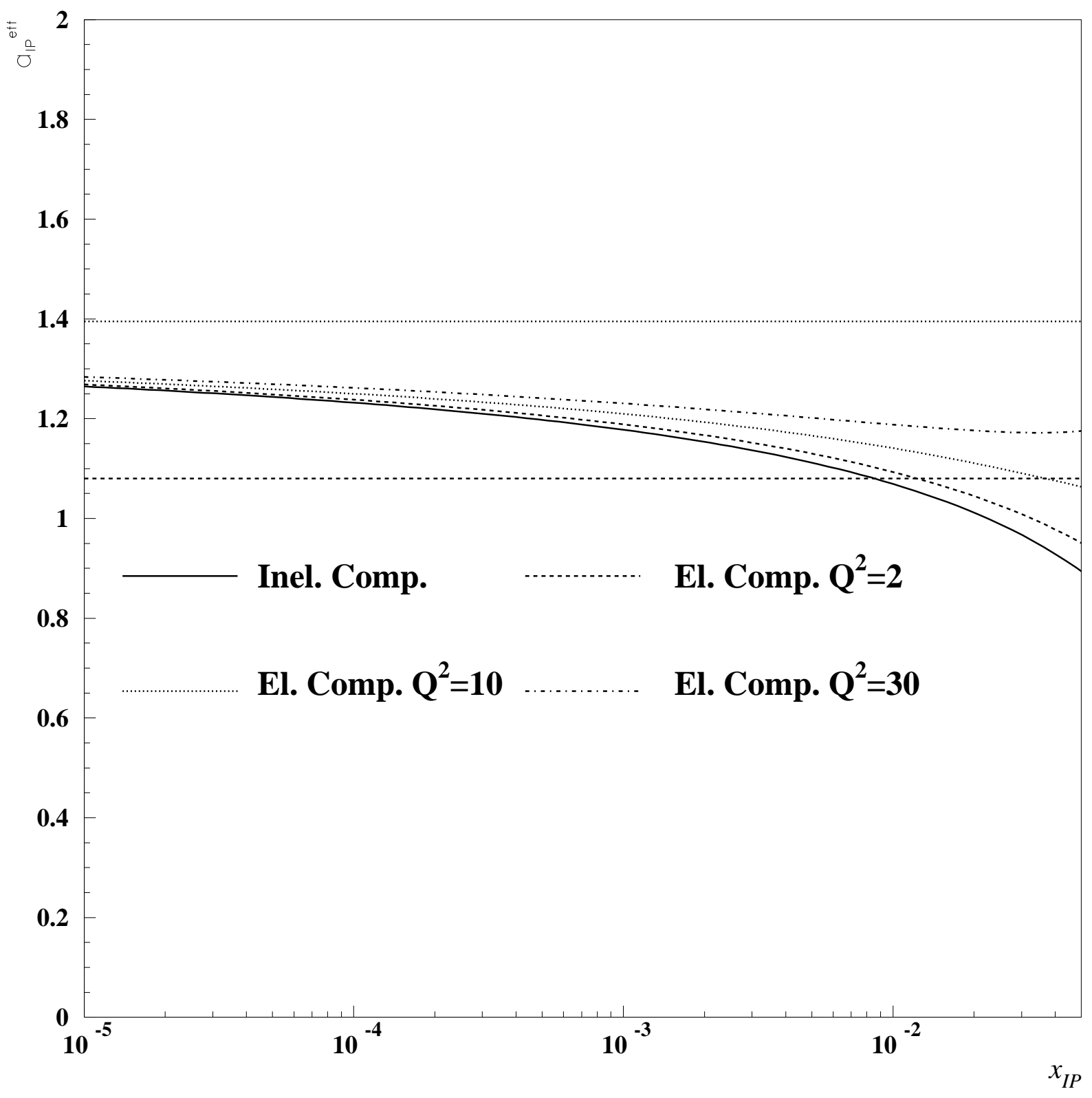

Figure 5 


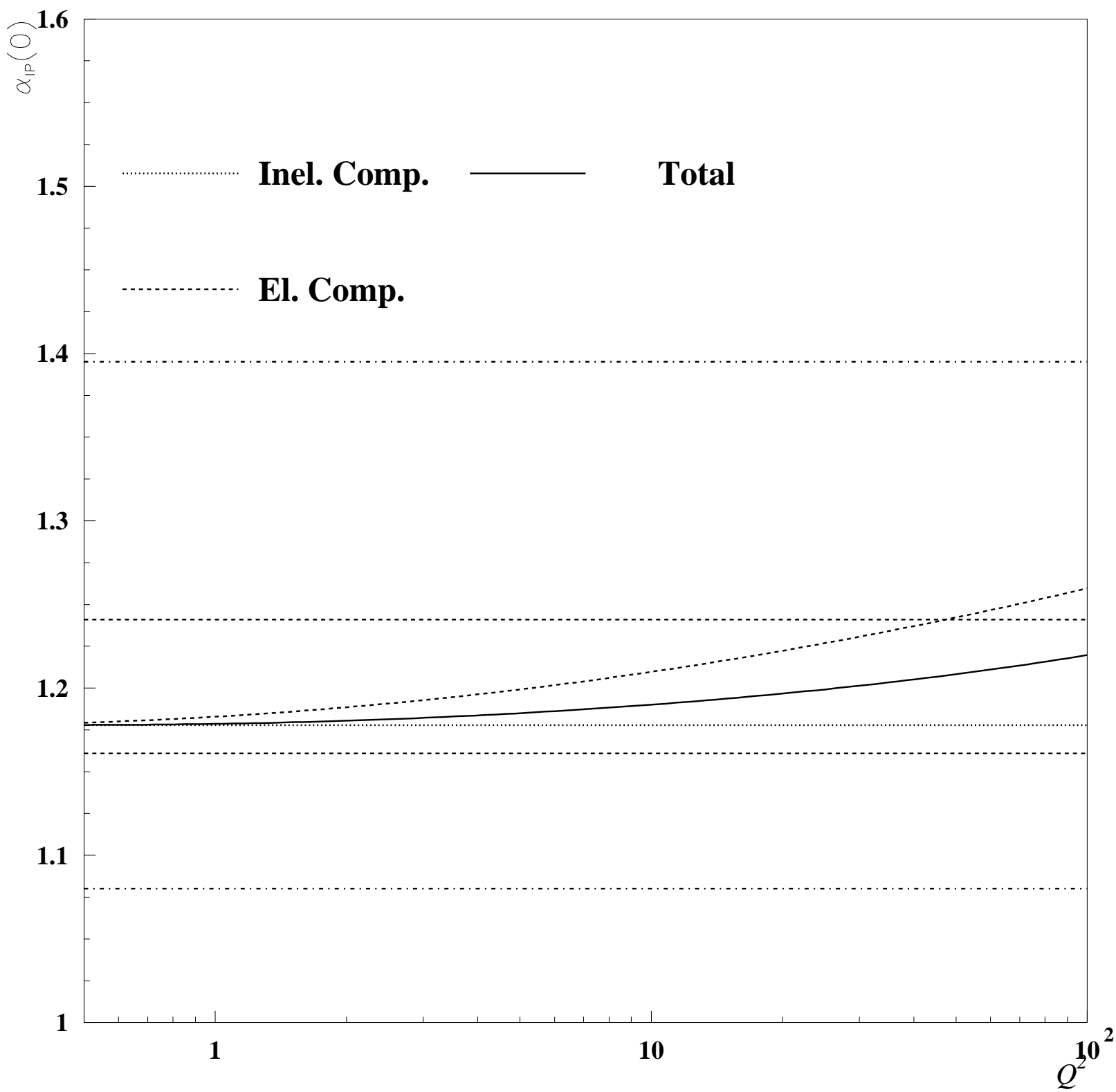

Figure 6 


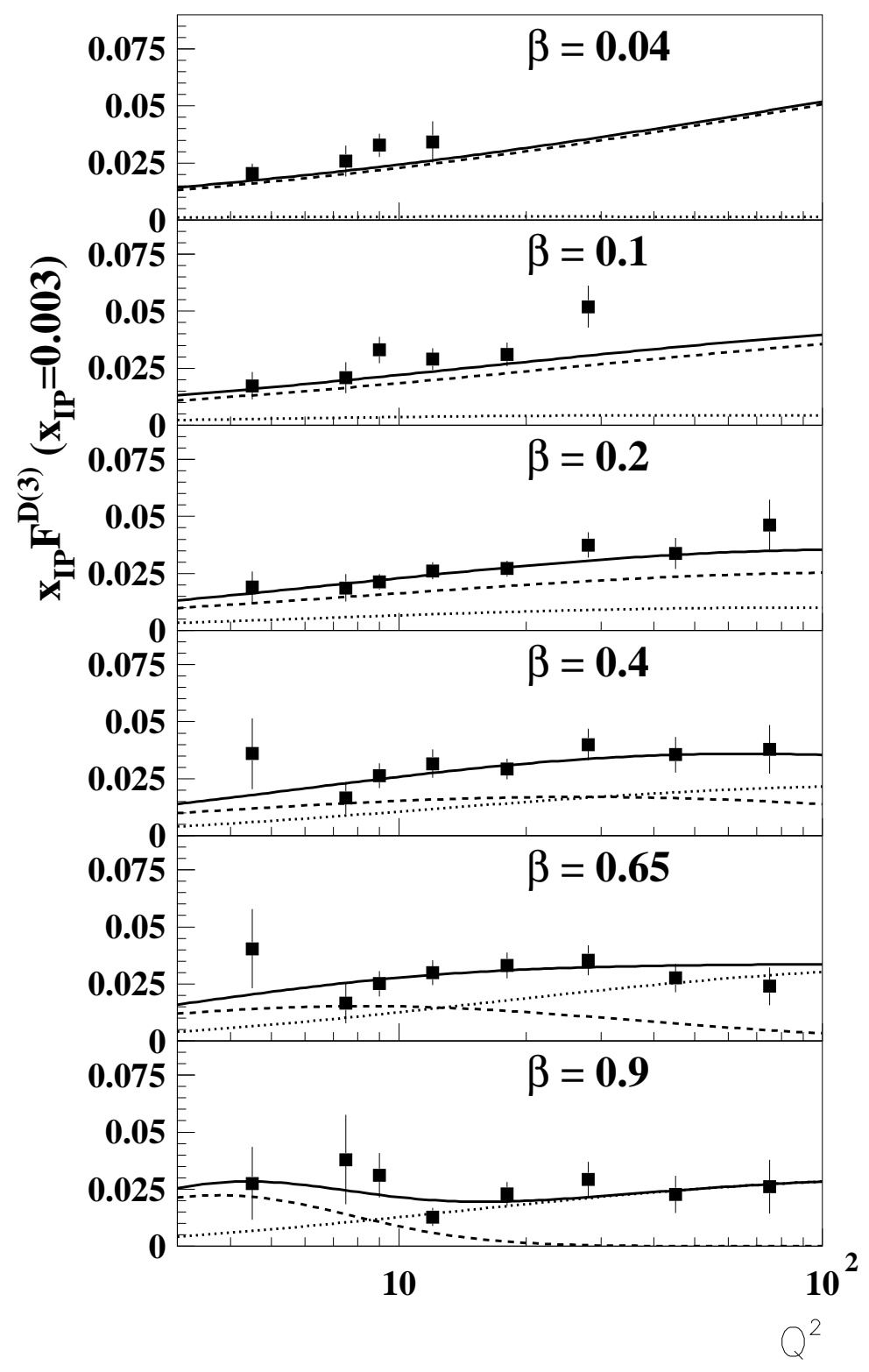

Figure 7 


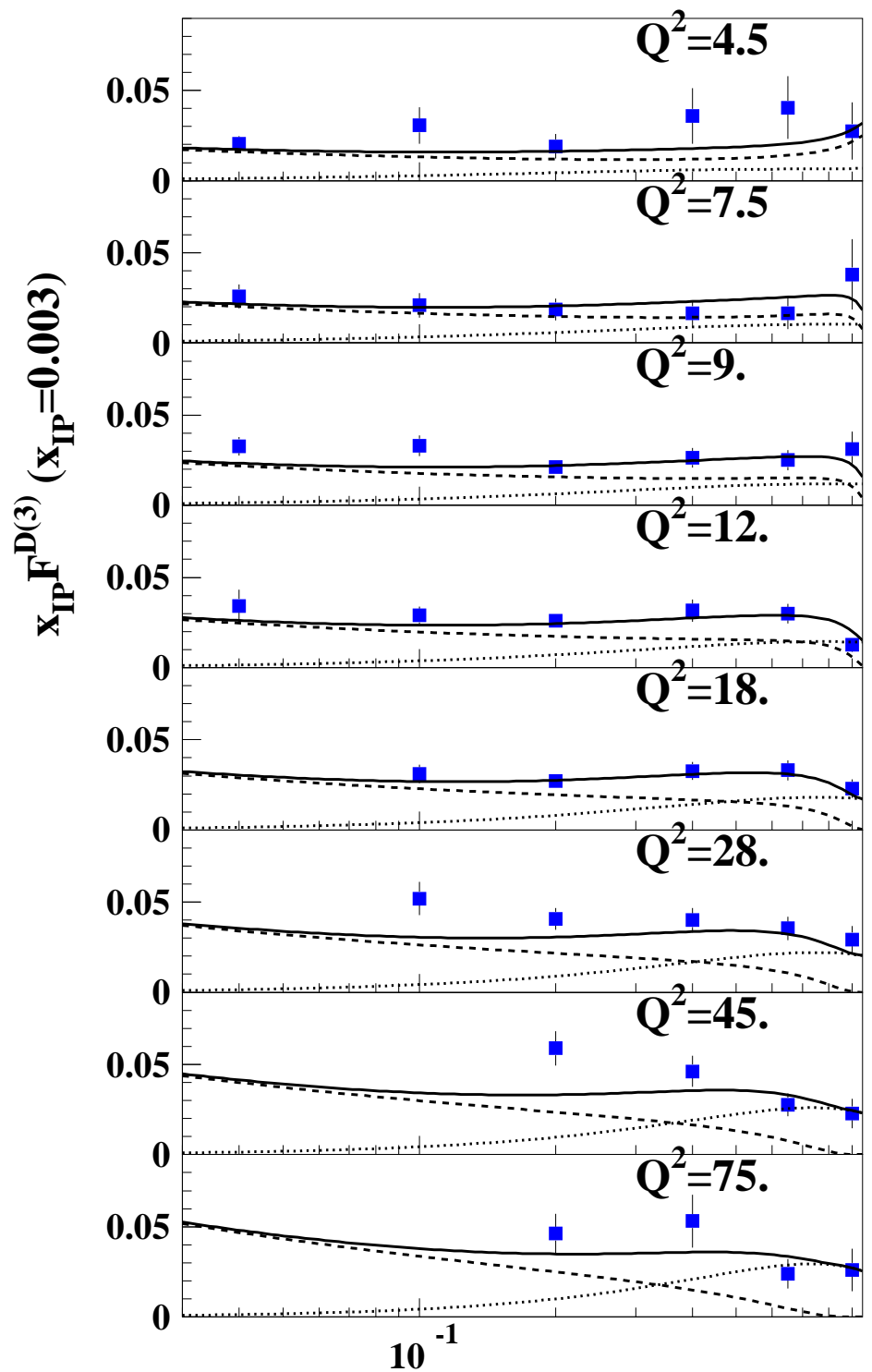

Figure 8 


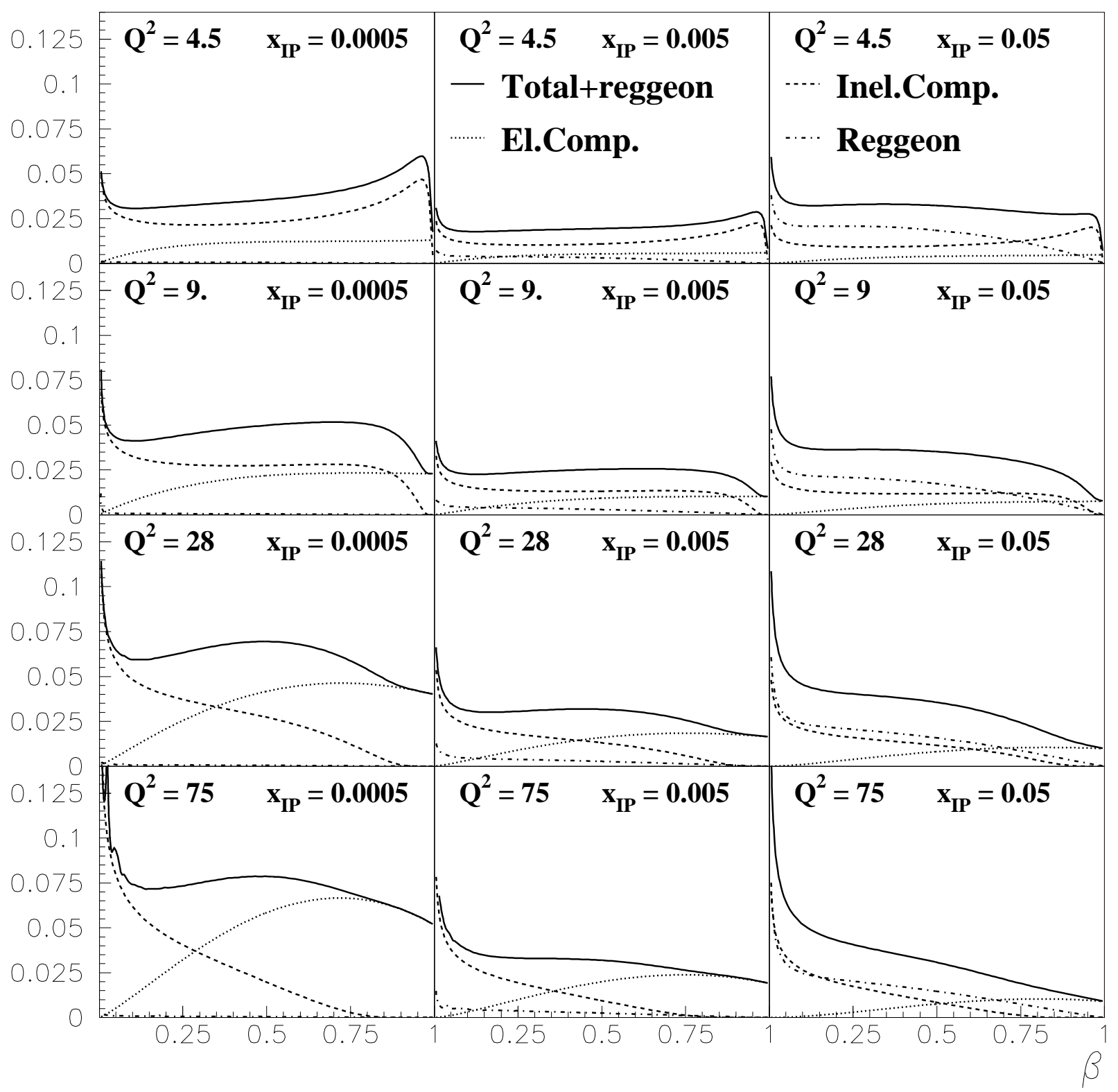

Figure 9 


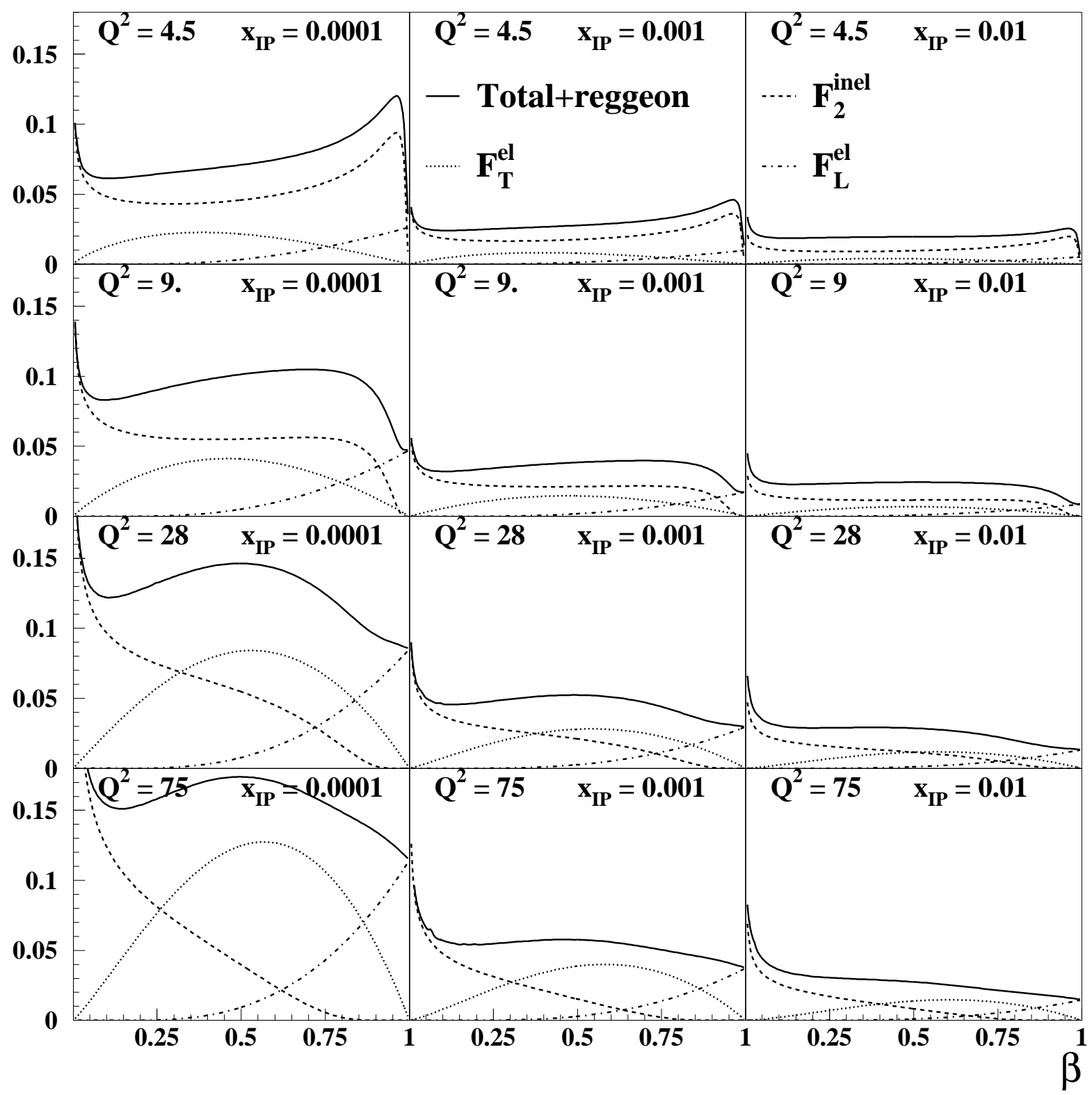

Figure 10 


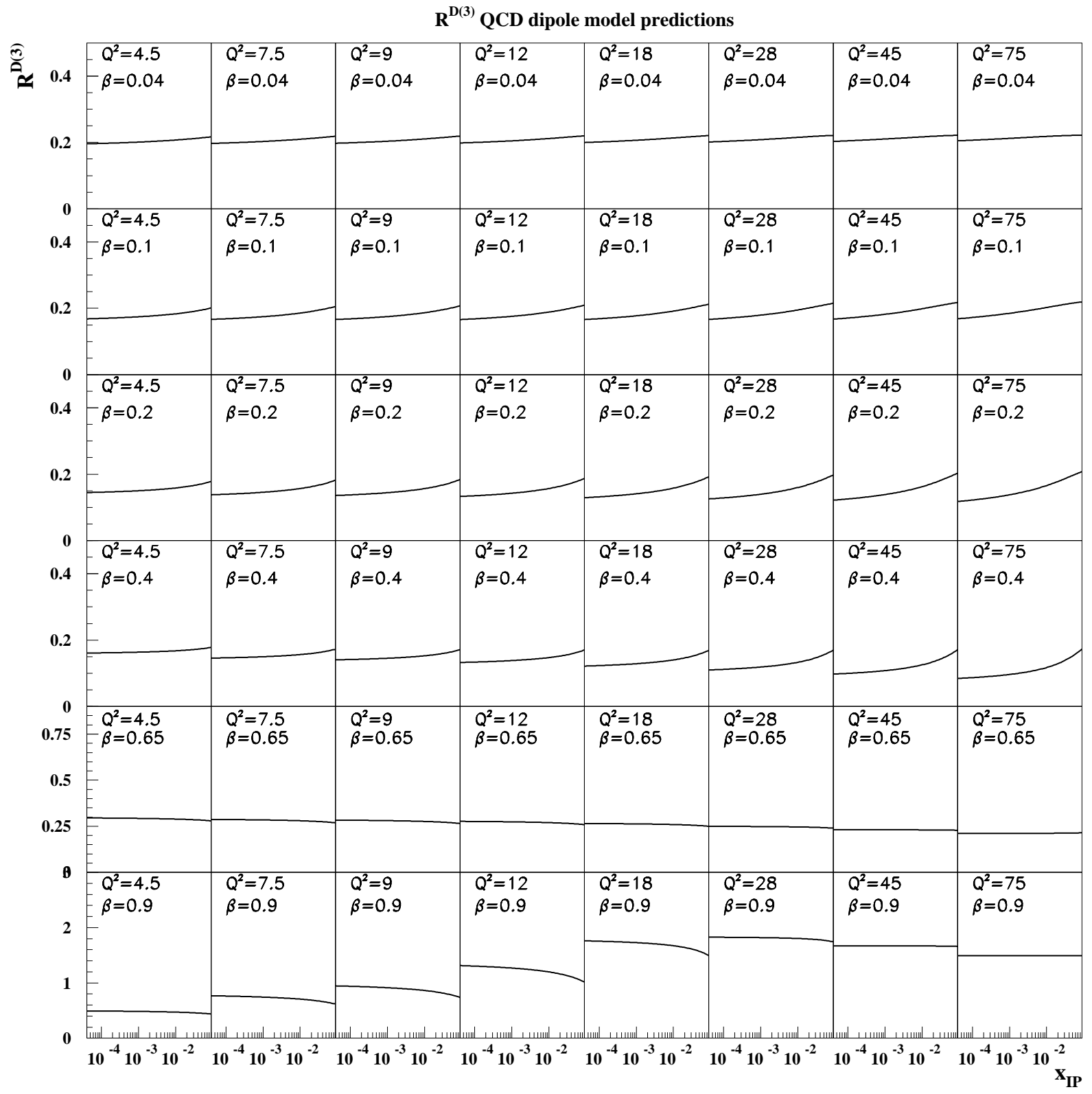

Figure 11 